\title{
Assessment of exposure to solvents among hairdressers: reliability of a classification scheme and questionnaire
}

\author{
W M Kersemaekers, N Verheijen, H Kromhout, N Roeleveld, G A Zielhuis
}

\begin{abstract}
Objectives-To assess the reliability of a classification scheme and interview questions to be used for retrospective expose assessment in a study on reproductive disorders among hairdressers. Based on the presence of an air cleaning device (yes or no) and setting waves (yes or no), this scheme divides hairdressers into groups with potentially high and low exposure to solvents. The reliability of this and other schemes was assessed. Also, the reliability of self reports on other job characteristics was evaluated.

Methods-The monitored hairdressers were interviewed one or two years after measurements were performed. Based on the interview information, hairdressers were classified into exposure groups according to the original and other classification schemes. Measured ethanol concentrations were compared between the classified exposure groups. Furthermore, the interview answers were compared with the registered information one to two years ago.
\end{abstract}

Results-Using self reports, the original scheme resulted in mean ethanol concentrations (used as indicator variable) of 11.8 and $7.4 \mathrm{mg} / \mathrm{m}^{3}$ for the high and low exposure groups, respectively. The resolution was slightly less than for the original classification based on observations (15.0 and $7.1 \mathrm{mg} / \mathrm{m}^{3}$ ). Surprisingly, the self reported presence of any ventilation device resulted in more contrast in mean exposure concentration between the groups (17.4 and $7.5 \mathrm{mg} / \mathrm{m}^{3}$, respectively). Hairdressers reported validly on salon characteristics such as the type of salon, the number of hairdressers that worked in the salon, and the presence of ventilation devices, but could not make a distinction between different kinds of ventilation devices. The numbers of customers and tasks performed were largely overreported, but most variables correlated significantly with the information registered during the measurements.

Conclusion-The self reported presence of any ventilation device is most predictive for the level of exposure to ethanol in the hairdressing salon. Questionnaire data on work characteristics should be treated with caution.

(Occup Environ Med 1998;55:37-42)
Keywords: exposure assessment; hairdressers; reliability

In occupational epidemiological studies, information on past exposure is often obtained by questionnaires. Some studies use self reported job titles as indicators of exposure. ${ }^{12}$ Other studies gather information on tasks or products used by individual workers, ${ }^{3-5}$ or use questions on exposure to specific chemicals. ${ }^{12}$ Recognition is growing that asking for specific tasks is most valid when the probability of exposure to specific substances is evaluated by experts. ${ }^{6-9}$ Also, information on job characteristics together with exposure data can be used to derive statistical models or grouping strategies to be used for retrospective exposure assessment in epidemiological studies. ${ }^{10}$

In a study on reproductive disorders among hairdressers, retrospective exposure to solvents is assessed through self reports of tasks and characteristics of the workplace in combination with a classification scheme which divides hairdressers into high and low exposure groups. ${ }^{11}$ This scheme was developed in an exposure assessment study in 1994 and 1995, in which ethanol concentrations were measured in several hairdressing salons. Ethanol concentrations were related to salon characteristics and tasks performed during the measurements to identify determinants of exposure. These data showed that based on two easily obtainable variables, setting waves (yes or no) and the presence of an air cleaning device (yes or no), hairdressers could be divided into high and low exposure groups which differed considerably in mean ethanol concentrations.

The same data on ethanol exposure were used to validate the classification scheme when applied to interview data of the monitored hairdressers one or two years later. The reliability of reporting on other relevant job characteristics was also investigated.

\section{Methods}

DESIGN

One or two years after the measurements the hairdressers were interviewed by telephone about their work at the time of the measurements. Based on the information from the interviews, the hairdressers were classified into exposure groups according to the original classification scheme. The reliability ${ }^{12}$ of this classification was assessed by comparing the earlier measured mean ethanol concentrations and their distributions between the classified exposure groups. Furthermore, the reliability of reporting on job characteristics was 
investigated by comparing the interview answers with the information registered one to two years previously.

\section{Data collection for the study of assessment of exposure ${ }^{11}$}

In the summer of 1994, personal eight hour time weighted average (TWA) measurements of ethanol were performed in 18 hairdressing salons in a rural area (59 hairdressers). The following year, in six of these salons measurements were repeated (29 hairdressers), and additional measurements were performed in nine salons in an urban area (26 hairdressers). In each salon, measurements were carried out on two consecutive days: Tuesday and Wednesday, or Thursday and Friday. Only the repeated measurements in the rural area were performed on four days of the week (Tuesday to Friday). ${ }^{11}$ Personal measurements were carried out with charcoal adsorbent tubes in the breathing zone. Each hairdresser registered information on standard registration forms about personal job characteristics during or at the end of the measurement day. This included information on the type of hairdresser (first, second, or junior), the number of customers, and the number of specific tasks (such as hair dyes, setting waves, hair sprays) performed during the measurement day. Salon characteristics were registered by an industrial hygienist, and included the presence of ventilation devices and number of hairdressers present in the salon. Table 3 of van Muiswinkel et al gives information on job characteristics in each measurement period and region. ${ }^{11}$

Data collection for the reliability study

We restricted the reliability study to hairdressing salons, and therefore excluded the hairdressing school (six hairdressers). The 26 hairdressing salons were approached by post and telephone in August 1996. After consent was given, the names and telephone numbers of the hairdressers who had been assessed were provided. These hairdressers were approached by telephone for a short computer assisted interview. Each hairdresser was approached at least four times at different times of the day. The interview included questions on their work in the hairdressing salon-such as the number of hours worked per week, the number of customers per week, the presence of ventilation devices in the salon, and the number of chemical tasks, such as setting waves and dyeswhich the hairdresser had performed per week. All questions referred to the month of the measurements (June 1994 or April 1995). When a hairdresser had participated in both periods (1994 and 1995), questions were asked on the most recent year, and only these measurement data were used in the analyses.

\section{Comparability of the data}

During the measurements, the number of tasks and measured ethanol concentrations were registered on the measurement day (eight hours), whereas in the telephone interviews we asked for the number of tasks a week, because we are interested in mean long term exposures in the epidemiological study. Therefore, we had to extrapolate the information from the measurements from days to weeks. If a hairdresser had been assessed on more than one day, the information was averaged over the measurement days, leading to a mean personal ethanol concentration and mean number of tasks performed per person per day. This mean ethanol concentration was used in the assessment of reliability of the classification scheme. The mean numbers of tasks and customers per day were divided by eight and multiplied by the reported number of hours the hairdresser had worked per week, leading to mean numbers of tasks and customers per week. During the measurements, the number of customers was registered per salon instead of per hairdresser. Therefore, this number was first divided by the number of hairdressers present in the salon during the measurements, assuming an equal distribution of number of customers per hairdresser.

\section{Assessment of reliability}

From the former workplace assessment study ${ }^{11}$ it seemed that the classification scheme based on water waving (yes or no) and the availability of an air cleaning device (yes or no) was most predictive for the level of exposure. The reliability of this original scheme was checked by grouping the population according to the interview information on these variables. Because hairdressers did not correctly report on the type of ventilation device (see results), alternative classification schemes were also explored. When applicable, two types of classification schemes were used. In both schemes, hairdressers were put in the high exposure group, when they had performed any wave settings in a salon without air cleaning. Initially, all remaining hairdressers were defined as low exposed. In some schemes, however, a further classification of the low exposure group took place: hairdressers were defined as low exposed when they did no wave settings in an air cleaned salon, and otherwise they were defined as medium exposed. For these classification groups, the measured mean and median ethanol concentrations and their distributions were compared, assuming the measured ethanol concentrations to be the gold standard.

The specificity and sensitivity of reporting on ventilation devices were used as indices of agreement, assuming that the information gathered during the measurements was closest to the truth (gold standard). Positive and negative predictive values were also calculated. For the number of wave settings and other tasks performed and for the number of customers and hairdressers in the salon, Spearman rank correlation coefficients were calculated. The distributions in percentiles (P10, P50, and P90) of the number of customers, hairdressers, and tasks were also compared between measurement and interview data to find out whether overestimation or underestimation occurred. Because the extrapolations of the measurement data from days to weeks may have introduced errors, especially in women 
Table 1 Participation of salons and hairdressers

\begin{tabular}{lll}
\hline & Salons $(n)$ & Hairdressers $(n)$ \\
\hline Salons sent a letter & 26 & $87^{\star}$ \\
Salons reached & 22 & $74^{\star}$ \\
Participating salons & 21 & 70 \\
Hairdressers reached & & 54 \\
Participating hairdressers & & 47
\end{tabular}

*Number of hairdressers who had been monitored in the salons approached and reached; these hairdressers were not approached themselves.

who had been assessed on one day only, additional analyses were performed for women who had been assessed on at least two days.

\section{Results}

Twenty six salons, in which 87 hairdressers had been monitored were approached by letter. Of the 22 salons that could be reached by telephone, one refused to participate. The remaining 21 salons agreed to participate and gave telephone numbers of 70 hairdressers. Of these, $54(77 \%)$ hairdressers were reached by telephone, of whom $47(87 \%)$ were willing to participate in the interview (table 1). Most hairdressers who could not be reached did not work in the specific salon any more and did not leave an address.

Most of the hairdressers $(77 \%)$ had worked between 20 and 40 hours a week, with a median of 36 hours a week. The number of days that the interviewed hairdressers participated in the measurements ranged from one to four days. Of the 47 hairdressers, 28 had been assessed on more than one day.

Mean ethanol concentrations and its distributions were similar between the population monitored and the subpopulation that participated in the interviews with a mean concentration of $7 \mathrm{mg} / \mathrm{m}^{3}$, and a P10, P50, and P90 of 2, 7 , and $25 \mathrm{mg} / \mathrm{m}^{3}$, respectively, in both populations.
Table 2 shows the mean ethanol concentrations and its distributions for several classification schemes. With self reports, the original classification scheme based on setting waves and air cleaning resulted in mean ethanol concentrations of 11.8 and $7.4 \mathrm{mg} / \mathrm{m}^{3}$ in the high and low exposure group, respectively. The distribution of ethanol concentrations overlapped considerably between these exposure groups. Setting waves did not improve the distinction between high and low exposure groups, the mean concentrations of ethanol based on air cleaning only were 11.4 and $7.4 \mathrm{mg} / \mathrm{m}^{3}$ in the high and low exposure groups, respectively. These resolutions are less than those from this scheme in the original population $\left(15.0 \mathrm{mg} / \mathrm{m}^{3}\right.$ and $\left.7.1 \mathrm{mg} / \mathrm{m}^{3}\right)$. Because hairdressers did not correctly report on the type of ventilation (see later), we included other ventilation devices in the classification scheme. The presence of any ventilation device (including air cleaning, forced ventilation, and air conditioning) seemed to be the best indicator for the level of exposure, with mean concentrations of 17.4 and $7.5 \mathrm{mg} / \mathrm{m}^{3}$ in the group without and with any ventilation device, respectively. Again, from interview data, setting waves did not improve the distinction between high and low exposure groups. With the scheme that included setting waves, however, the low exposure group could be further divided into medium and low exposure, by which more contrast in mean ethanol concentrations was achieved between high and low exposure groups $\left(17.4\right.$ and $5.1 \mathrm{mg} / \mathrm{m}^{3}$, respectively), with little overlap in the distributions between these groups $\left(\mathrm{P} 0_{\text {low }}=8.8\right.$ and $\left.\mathrm{P} 10_{\text {high }}=8.2 \mathrm{mg} / \mathrm{m}^{3}\right)$. However, most hairdressers were classified in the medium exposure group, of which the distribution of ethanol concentration largely overlaps with those of the

Table 2 Distribution of measured ethanol concentrations within groups of hairdressers, classified by the presence of ventilation devices and wave settings according to interview data

\begin{tabular}{|c|c|c|c|c|c|}
\hline \multirow[b]{2}{*}{ Classification schemes } & \multirow[b]{2}{*}{$n^{*}$} & \multicolumn{4}{|c|}{ Ethanol concentration $\left(\mathrm{mg} / \mathrm{m}^{3}\right)$} \\
\hline & & Meant & 10th percentile & 50th percentile & 90th percentile \\
\hline \multicolumn{6}{|l|}{ Wave setting: } \\
\hline Yes (high) & 41 & 9.6 & 2.4 & 7.3 & 24.7 \\
\hline No (low) & 4 & 5.1 & 3.2 & 4.3 & 8.8 \\
\hline \multicolumn{6}{|l|}{ Air cleaning: } \\
\hline No (high) & 20 & 11.4 & 3.2 & 7.8 & 26.2 \\
\hline Yes (low) & 23 & 7.4 & 2.1 & 5.2 & 14.4 \\
\hline \multicolumn{6}{|c|}{ Wave setting and air cleaning (original scheme): $\ddagger$} \\
\hline High & 19 & 11.8 & 2.7 & 8.2 & 26.9 \\
\hline Low & 25 & 7.4 & 2.1 & 5.2 & 14.4 \\
\hline \multicolumn{6}{|c|}{ Air cleaning or forced ventilation: } \\
\hline No (high) & 18 & 10.9 & 2.7 & 7.8 & 25.4 \\
\hline Yes (low) & 29 & 8.1 & 2.1 & 5.9 & 24.7 \\
\hline \multicolumn{6}{|c|}{ Wave setting and air cleaning or forced ventilation: $\ddagger$} \\
\hline High & 17 & 11.3 & 2.7 & 8.2 & 25.4 \\
\hline Low & 29 & 8.1 & 2.1 & 5.9 & 24.7 \\
\hline \multicolumn{6}{|l|}{ Any ventilation device: } \\
\hline No (high) & 8 & 17.4 & 8.2 & 15.6 & 36.0 \\
\hline Yes (low) & 39 & 7.5 & 2.2 & 5.2 & 14.7 \\
\hline \multicolumn{6}{|c|}{ Wave setting and any ventilation device: $\ddagger$} \\
\hline High & 8 & 17.4 & 8.2 & 15.6 & 36.0 \\
\hline Low & 39 & 7.5 & 2.2 & 5.2 & 14.7 \\
\hline \multicolumn{6}{|c|}{ Wave setting and any ventilation device: $\ddagger$} \\
\hline High & 8 & 17.4 & 8.2 & 15.6 & 36.0 \\
\hline Medium & 33 & 7.7 & 2.2 & 5.9 & 14.7 \\
\hline Low & 4 & 5.1 & 3.2 & 4.3 & 8.8 \\
\hline
\end{tabular}

^Total number of observations per model may differ because hairdressers with a missing value on one of the variables sometimes can and sometimes cannot be divided into an exposure group

†Arithmetic mean of individual mean exposures .

$\ddagger$ High=setting waves in a salon without the specified ventilation device; low=all other combinations of answers to questions. 
Table 3 Reliability of reporting on ventilation devices in the hairdressing salon

\begin{tabular}{|c|c|c|c|c|c|c|c|}
\hline \multirow[b]{2}{*}{ Interviews } & & \multicolumn{2}{|c|}{ Measurements } & \multirow{2}{*}{$\begin{array}{l}P V+ \\
(\%)\end{array}$} & \multirow{2}{*}{$\begin{array}{l}P V- \\
(\%)\end{array}$} & \multirow{2}{*}{$\begin{array}{l}\text { Sensitivity } \\
(\%)\end{array}$} & \multirow{2}{*}{$\begin{array}{l}\text { Specificity } \\
(\%)\end{array}$} \\
\hline & & Yes & No & & & & \\
\hline \multirow[t]{2}{*}{ Any ventilation device } & Yes & 30 & 9 & 77 & 100 & 100 & 47 \\
\hline & No & 0 & 8 & & & & \\
\hline \multirow[t]{2}{*}{ Forced ventilation or air cleaning device } & Yes & 17 & 12 & 59 & 89 & 89 & 57 \\
\hline & No & 2 & 16 & & & & \\
\hline \multirow[t]{2}{*}{ Forced ventilation ${ }^{\star}$} & Yes & 1 & 1 & 50 & 64 & 8 & 97 \\
\hline & No & 12 & 29 & & & & \\
\hline \multirow{2}{*}{ Air cleaning ${ }^{\star}$} & Yes & 7 & 16 & 30 & 100 & 100 & 56 \\
\hline & No & 0 & 20 & & & & \\
\hline
\end{tabular}

^Hairdressers were excluded who did not know whether forced ventilation or air cleaning devices were present $(n=4)$.

high and low exposure groups $\left(\mathrm{P} 90_{\text {low }}=8.8\right.$ and $\left.\mathrm{P} 10_{\text {high }}=8.2 \mathrm{mg} / \mathrm{m}^{3}\right)$. However, most hairdressers were classified as being in the medium exposure group, in which the distribution of ethanol concentration largely overlaps that of the high and low exposure groups (table 2).

The reliability of self reports on other job characteristics, and the type of salon was good, only two hairdressers reported having worked in a mixed salon instead of a women's salon. Also, most of the hairdressers (83\%) correctly remembered whether they were first, second, or junior hairdresser during the measurements.

Table 3 shows the results on ventilation devices. All the hairdressers who had worked in a salon with forced general ventilation, an air cleaning device, or with an air conditioner reported so in the telephone interview (sensitivity $=100 \%$ ). However, nine hairdressers reported having worked in a salon with one of these ventilation devices, when that was not the case (specificity $=47 \%$ ). Positive and negative predictive values (PVs) on any ventilation device were relatively high $(77 \%$ and $100 \%)$. Most of the hairdressers who worked in a salon with forced ventilation or air cleaning reported so (sensitivity $=89 \%$ ), but there was much overreporting (specificity $=57 \%$ ). It seemed to be difficult for hairdressers to make a distinction between forced ventilation and air cleaning, with air cleaning devices more often reported. Ten of the 14 hairdressers who had worked in a salon with forced ventilation in the salon reported that they had worked in a salon with air cleaning. This may account for the low sensitivity $(8 \%)$ for forced ventilation and the low positive predictive value $(30 \%)$ and specificity $(56 \%)$ for air cleaning devices. Hairdressing salons with more than three hairdressers always had a ventilation device, but this was the case in only $33 \%$ of the salons with two or three hairdressers.

Table 4 shows the results for the number of hairdressers and customers in the salon, and the number of tasks performed each week. For all variables, considerable overestimation occurred in the interviews compared with the data registered during the measurements, except for the number of hairdressers who worked in the salon simultaneously. Correlation coefficients were highest for the number of hairdressers (0.64) and customers (0.63) in the salon, whereas the correlation coefficients for the number of tasks were lower (0.21-0.49). If only hairdressers who were assessed on more than one day were included, differences in the distribution (P10, P50, and P90) between interview data and data registered during the measurements did not decrease. However, correlation coefficients increased for most variables. Correlation coefficients were 0.76 for the number of hairdressers in the salon and 0.83 for the number of customers a week. For the number of hair dyes the correlation coefficient increased from 0.21 to $0.41(\mathrm{P}=0.03)$, and for sweeping and the number of waves set the correlation

Table 4 Reliability of reporting on number of hairdressers in the salon and mean number of customers and tasks performed per week

\begin{tabular}{|c|c|c|c|c|c|c|c|c|c|}
\hline & \multirow[b]{2}{*}{$n$} & \multirow[b]{2}{*}{ Mean } & \multicolumn{3}{|c|}{ Percentile } & \multirow[b]{2}{*}{$R^{\star}$} & \multirow[b]{2}{*}{ Pvalue } & \multirow[b]{2}{*}{$R^{\star} t$} & \multirow[b]{2}{*}{ P value } \\
\hline & & & $10 \%$ & $50 \%$ & $90 \%$ & & & & \\
\hline \multicolumn{10}{|c|}{ Hairdressers in salon: } \\
\hline Measurement & 47 & 4 & 2 & 4 & 5 & 0.64 & 0.00 & 0.76 & 0.00 \\
\hline Interview & 47 & 4 & 2 & 4 & 7 & & & & \\
\hline \multicolumn{10}{|c|}{ Customers a week: } \\
\hline Measurement & 40 & 35 & 18 & 35 & 52 & 0.63 & 0.00 & 0.83 & 0.00 \\
\hline Interview & 40 & 59 & 37 & 55 & 88 & & & & \\
\hline \multicolumn{10}{|l|}{ Hair dyes: } \\
\hline Measurement & 45 & 2 & 0 & 0 & 5 & 0.21 & 0.16 & 0.41 & 0.03 \\
\hline Interview & 45 & 6 & 1 & 5 & 15 & & & & \\
\hline \multicolumn{10}{|l|}{ Wave setting: } \\
\hline Measurement & 45 & 3 & 0 & 0 & 8 & 0.48 & 0.00 & 0.56 & 0.00 \\
\hline Interview & 45 & 11 & 1 & 8 & 30 & & & & \\
\hline \multicolumn{10}{|l|}{ Permanent waves: } \\
\hline Measurement & 44 & 4 & 0 & 2 & 7 & 0.29 & 0.06 & 0.14 & 0.51 \\
\hline Interview & 44 & 7 & 3 & 6 & 15 & & & & \\
\hline \multicolumn{10}{|l|}{ Hair spray use: } \\
\hline Measurement & 31 & 12 & 0 & 12 & 21 & 0.45 & 0.01 & 0.32 & 0.15 \\
\hline Interview & 31 & 34 & 12 & 30 & 60 & & & & \\
\hline \multicolumn{10}{|l|}{ Sweeping: } \\
\hline Measurement & 44 & 32 & 10 & 29 & 54 & 0.49 & 0.00 & 0.56 & 0.00 \\
\hline Interview & 44 & 48 & 12 & 45 & 80 & & & & \\
\hline
\end{tabular}

*Spearman's correlation coefficients.

tOnly hairdressers who had been assessed more than once were included $(n=28)$. 
coefficient was 0.56 among women who had been assessed on more than one day $(\mathrm{P}=0.00)$. Only for the number of permanent waves and the number of hair sprays did the correlation coefficients decrease. Surprisingly, for these variables correlation coefficients were highest when restricting the analyses to women who had been measured on one day $\left(R_{\text {permanents }}=0.44\right.$, $\mathrm{R}_{\text {hairspray }}=0.61$ ). Of the 46 hairdressers who reported which brand of hair spray they used most often, $34(72 \%)$ mentioned the brand which was registered during the measurements.

\section{Discussion}

This study provides relevant information on the reliability of a classification scheme and interview questions to be used for exposure assessment in a study on reproductive disorders among hairdressers, but it has some methodological limitations.

Only $54 \%$ of the hairdressers who were assessed were contacted for the telephone interview. However, for measured ethanol concentration this interviewed population was representative of the total. Therefore, the classification scheme described should be applicable to the interviewed subpopulation and could thus be validly checked. Furthermore, it is unlikely that the reliability of reporting on the questions differs between the hairdressers who participated in the interviews and those who did not.

With self reports, the original classification scheme resulted in mean ethanol concentrations of 11.8 and $7.4 \mathrm{mg} / \mathrm{m}^{3}$ for the high and low exposure group, respectively. The resolution was slightly less than for the original classification based on observations (15.0 and $\left.7.1 \mathrm{mg} / \mathrm{m}^{3}\right) .{ }^{11}$ This can partly be explained by the fact that we used mainly data from the second measurement period in which the resolution in exposure concentrations was lower. ${ }^{11}$ Surprisingly, the interview information on the presence of any ventilation devices resulted in more contrast in mean exposure between the groups (15.0 and $7.1 \mathrm{mg} / \mathrm{m}^{3}$, respectively). This is probably due to the large number of incorrect self reports on type of ventilation. Furthermore, with self reports, no improvement was reached by setting waves in both the original scheme and the adjusted scheme based on the presence of any ventilation device and two exposure categories (table 2). This might be explained by the small contrast in this interview variable: only four hairdressers did not set any waves each week. This discrepancy between measurements and interviews in number of hairdressers who did not set waves may be due to the difference in registration per day during the measurements and per week during the interviews. When classifying the population into three exposure groups, however, the variable setting waves did improve the contrast between high and low. But as most hairdressers would have had medium exposure according to this scheme, restricting the comparison to high and low exposure groups would lead to too much loss of power. The medium exposure group is too diverse for relevant salon charac- teristics, as it includes salons with and without presence of ventilation devices and with and without setting waves.

For salon characteristics-such as the type of salon-the number of hairdressers who worked in a salon and presence of ventilation devicesthe gold standard is assumed to be appropriate, as these items were recorded by the industrial hygienists during the measurements. These variables were reported reliably in the interviews. Our data show that hairdressers knew whether a ventilation device was present (sensitivity $=100 \%$ ), but presence of one was overreported considerably (specificity $=47 \%$ ). Hairdressers seemed to be unable to make the distinction between forced ventilation and air cleaning, and preferentially reported on the presence of an air cleaning device. The hypothesis that more hairdressers in a salon will yield higher levels of exposure is refuted by our data, which showed that all salons with more than three hairdressers had a ventilation device; this seemed to be the most important determinant of the level of exposure.

The number of customers and the number of chemical tasks performed seemed to be overreported in the interview. Correlation coefficients were relatively high for the number of customers, but moderate correlations were found for the number of chemical tasks performed in a week. In validating the interview data on these personal characteristics, the appropriateness of the gold standard should be considered.

During the measurements, hairdressers themselves registered the number of customers and chemical tasks, which might have introduced errors. Furthermore, this information was registered per day, but was reported per week in the interviews. This may have led to errors, especially on tasks that are not performed every day (most of the chemical tasks). This error in the gold standard is expected to decrease with the number of measurement days of a specific hairdresser, which also appeared from higher correlation coefficients among women who had been measured on more than one day. However, overestimation of the number of customers and tasks occurred to the same extent among women who had been measured once or on more than one day. This overestimation might be explained by the fact that measurements were performed from Tuesday to Friday, whereas in the interviews hairdressers reported on weeks, including Mondays, Saturdays, and shopping nights. The number of customers was highest on Fridays, ${ }^{11}$ and would be expected to also be high on Saturdays and shopping nights. We did not take into account this day to day variance in the extrapolation of the measurement data from days to weeks, because day to day variance differed for each hairdresser and exposure variable. For most variables, this might have led to the gold standard being too low, especially for women who have not been assessed on Fridays. However, separate analyses among women who had been assessed on Fridays and on other days did not show consistent differences in correlation coefficients or the amount of overestimation. However, there were small numbers in 
this subanalysis. Also, the amount of overestimation of the number of customers was similar between full time ( $>30$ hours a week) and part time workers ( $\leqslant 30$ hours a week), but the part time workers may have worked at times when the number of customers peaked, which could have led to more overestimation. If mean numbers of customers per week were calculated under the assumption that Fridays and Saturdays are similar, this resulted in an average number of $38,{ }^{11}$ instead of 34 from our calculation, whereas the mean number of customers according to the interviews was 58 . Therefore, it is concluded that the method of extrapolation from days to weeks does not entirely account for the overestimations found. Therefore, careful interpretation is needed when exact numbers of performed tasks are used for estimation of exposures, or when dose-response relations are translated to preventive measures.

In an earlier study in The Netherlands on menstrual disorders among hairdressers, ${ }^{4}$ exposure to hairdressers' chemicals was assessed by self reported questionnaires. In this study, the reported number of performed tasks was comparable with those reported in our telephone interviews. In the American study of John et $a \bar{l}$ on spontaneous abortions among cosmetologists, the number of self reported tasks and customers was somewhere in between our numbers reported in the interviews and the measurements. ${ }^{11}$ The mean number of hours worked was about 36 a week in both studies, and thus comparable with our study. In the study of Blatter and Zielhuis, ${ }^{4}$ no doseresponse relation was found, but John et $a l^{5}$ found increasing odds ratios with the number of chemical services performed. Because all data were collected in the same way in that study, this dose-response relation may be valid. However, when translating these results into preventive measures, the probability of overestimation of number of tasks should be taken into account.

The present study shows that the reporting on one easily obtainable variable, the presence of any ventilation device, was more predictive for the level of exposure to ethanol than the original scheme based on air cleaning and set- ting waves. It seems plausible to assume that presence of any ventilation devices will also be predictive for the level of exposure to other solvents or even other volatile agents. The number of hairdressers in the salon was reported reliably, and the number of customers a week was overestimated, but reports correlated fairly well with the information from the measurements. The interview questions on chemical tasks of hairdressers were overreported considerably by the hairdressers, but most variables correlated significantly with information registered during the measurements. Application of these results to the epidemiological study on reproductive disorders among hairdressers will lead to careful interpretation on the reported number of performed tasks, and a classification scheme based on the presence of ventilation should be used instead of a scheme based on the presence of an air cleaning device and the setting of waves.

This work was supported by a grant from The Netherlands Organization of Scientific Research (900-563-065).

1 Decouflé P, Murphy C, Drews CD, Yeargin-Allsopp M. Mental retardation in ten-year-old children in relation to their mother's employment during pregnancy. $A m$ F Ind Med 1993;24:567-86.

2 Roeleveld N. Mental retardation and parental occupation: an explorative epidemiologic study [thesis]. Nijmegen, The Netherlands: University of Nijmegen, 1991.

3 Cordier S, Ha MC, Ayme S, Goujard J. Maternal occupational exposure and congenital malformations. Scand $\mathcal{F}$ Work Environ Health 1992;18:11-7.

4 Blatter BM, Zielhuis GA. Menstrual disorders due to chemical exposure among hairdressers. Occup Med 1993; 43:105-6.

5 John EM, Savitz DA, Shy CM. Spontaneous abortions among cosmetologists. Epidemiology 1994;5:147-54.

6 Teschke K, Kennedy SM, Olshan AF. Effect of different questionnaire formats on reporting of occupational expoquestionnaire formats on reporting of
sures. Am f Ind Med 1994;26:327-37.

7 Gerin $M$, Siemiatycki J, Kemper H, Begin D. Obtaining occupational exposure histories in epidemiologic caseoccupational exposure histories in epidem
control studies. $\mathcal{F}$ Occup Med 1985;27:420-6.

control studies. F Occup Med 1985;27:420-6.
8 Fritschi L, Siemiatycki J, Richardson L. Sef-assessed versus Fritschi L, Siemiatycki J, Richardson L. Sef-assessed versus
expert-assessed occupational exposures. Am $\mathcal{f}$ Epidemiol 1996;144:521-7.

9 Stewart PA, Stewart WF. Occupational case-control studies: two recommendations for exposure assessment. Am f Ind Med 1994;26:313-26.

10 Kromhout H. From eyeballing to statistical modelling. Methods for assessment of occupational exposure [thesis]. Wageningen, The Netherlands: University of Wageningen, 1994.

11 van Muiswinkel WJ, Kromhout H, Onos T, Kersemaekers WM. Monitoring and modelling of exposure to ethanol in hairdressing salons. Ann Occup Hyg 1997;41:235-47.

12 Armstrong BK, White E, Saracci R. Principles of exposure measurement in epidemiology. Oxford: Oxford University Press, 1992. 